\title{
UNIQUENESS OF WEAK SOLUTIONS TO A MODEL OF ELECTRO-KINETIC FLUID*
}

\author{
JISHAN FAN $^{\dagger}$ AND HONGJUN GAO $\ddagger$
}

\begin{abstract}
In this paper we prove the uniqueness of weak solutions to a model of electro-kinetic fluid which consists of a momentum equation together with transport equations of charges. Our result is new in that it holds even when the momentum vanishes. The existence of weak solutions has been proved in [P.Biler, W. Hebisch and T. Nadzieja, Nonlinear Anal. TMA, 23, 1189-1209, 1994], [R. Ryham, C. Liu and Z-Q. Wang, Preprint, 2005], [R. Ryham, arXiv: 0810.2064 v1 (math.AP) 12, Oct 2008].
\end{abstract}

Key words. Uniqueness, weak solution, electro-kinetic fluid.

AMS subject classifications. 35Q30.

\section{Introduction}

The equations governing the hydrodynamic transport of binary diffuse charge densities are $[1,2,3]$,

$$
\begin{gathered}
u_{t}+u \cdot \nabla u+\nabla \pi-\Delta u=\varepsilon \Delta \varphi \nabla \varphi, \\
\operatorname{div} u=0, \\
n_{t}+u \cdot \nabla n=\nabla \cdot(\nabla n-n \nabla \varphi), \\
p_{t}+u \cdot \nabla p=\nabla \cdot(\nabla p+p \nabla \varphi), \\
\varepsilon \Delta \varphi=n-p, \quad \text { in } \quad Q_{T}:=(0, T) \times \Omega
\end{gathered}
$$

with boundary and initial conditions

$$
\begin{gathered}
\left.u\right|_{\partial \Omega}=0, \\
\left.(\nabla n-n \nabla \varphi) \cdot \nu\right|_{\partial \Omega}=\left.(\nabla p+p \nabla \varphi) \cdot \nu\right|_{\partial \Omega}=\left.\nabla \varphi \cdot \nu\right|_{\partial \Omega}=0, \\
\left.(u, n, p)\right|_{t=0}=\left(u_{0}, n_{0}, p_{0}\right), \operatorname{div} u_{0}=0, \text { in } \Omega .
\end{gathered}
$$

Here $\Omega \subseteq \mathbb{R}^{d}(d=2,3)$ is a bounded domain with smooth boundary $\partial \Omega, \nu$ is the unit outward normal to $\partial \Omega$.

The first equation (1.1) is the linear momentum equation of incompressible flow, and (1.1) and (1.2) are Navier-Stokes equation with the Lorentz force $\varepsilon \Delta \varphi \nabla \varphi$. u is the velocity field and $\pi$ is the pressure.

Equations (1.3), (1.4), and (1.5) are known as the electro-chemical equations [4, $5]$ or semiconductor equations $[6,7,8,9]$, and electrorheological systems $[2,3,10$, $11,12]$ when formally setting $u=0$. Here, $n$ and $p$ are the charge densities of a negatively and positively charged species, respectively, hence the sign difference in

${ }^{*}$ Received: February 6, 2009, accepted (in revised version): March 13, 2009. Communicated by Chun Liu.

†Institute of Mathematics, School of Mathematics and Computer Sciences, Nanjing Normal University, Nanjing 210097, P. R. China, and Department of Applied Mathematics, Nanjing Forestry University, Nanjing 210037, P. R. China (fanjishan@njfu.edu.cn).

${ }_{\ddagger}^{\ddagger}$ Institute of Mathematics, School of Mathematics and Computer Sciences, Nanjing Normal University, Nanjing 210097, P. R. China, and Center of Nonlinear Science, Nanjing University, Nanjing 210093, P. R. China (gaohj@njnu.edu.cn). 
front of the convective term in either equations, $\varphi$ is the electrostatic potential, and $\varepsilon$ is a small parameter, known as the Debye length, related to vacuum permittivity and characteristic charge density.

Very recently, Ryham, Liu and Wang [13] studied the system (1.1)-(1.8) and proved the global existence of weak solutions when $u_{0} \in L^{2}(\Omega)$ and $n_{0}, p_{0} \in L^{2}(\Omega)$ and studied the qualitative properties of solutions to the corresponding 1-d stationary problem with the Dirichlet boundary condition as $\varepsilon$ goes to zero in [14].

Kurokiba and Ogawa [6] considered the semiconductor device equation (1.3), (1.4) and (1.5) when formally setting $u=0$ and $\Omega=\mathbb{R}^{d}(d \geq 2)$ and proved the existence and uniqueness of weak solutions with $L^{p}$ initial data $\left(n_{0}, p_{0}\right)$ when $p=\frac{d}{2}(d \geq 4)$, $p>\frac{d}{2}(d=3)$, and $p \geq \frac{4}{3}(d=2)$.

Note that the system (1.1)-(1.5) holds its form under the scaling $(u, \pi, n, p, \varphi) \rightarrow$ $\left(u_{\lambda}, \pi_{\lambda}, n_{\lambda}, p_{\lambda}, \varphi_{\lambda}\right):=\left(\lambda u\left(\lambda^{2} t, \lambda x\right), \lambda^{2} \pi\left(\lambda^{2} t, \lambda x\right), \lambda^{2} n\left(\lambda^{2} t, \lambda x\right), \lambda^{2} p\left(\lambda^{2} t, \lambda x\right), \varphi\left(\lambda^{2} t, \lambda x\right)\right)$. Under this scaling, the space $L^{\theta}\left(0, T ; L^{p}\right)$ is invariant for $u$ when $\frac{2}{\theta}+\frac{d}{p}=1$ and the space $L^{\theta}\left(0, T ; L^{p}\right)$ is invariant for $(n, p)$ when $\frac{2}{\theta}+\frac{d}{p}=2$. Furthermore, $L^{d}$ for $u_{0}$ and $L^{d / 2}$ for $\left(n_{0}, p_{0}\right)$ are invariant spaces under this scaling.

The aim of this paper is to study the uniqueness of weak solutions in critical spaces. We will prove the following results.

THEOREM 1.1. Let $\left(n_{0}, p_{0}\right) \in L \log L, u_{0} \in L^{2}, n_{0}, p_{0} \geq 0$ in $\Omega \subseteq \mathbb{R}^{2}$ and $\int_{\Omega} \varphi \mathrm{d} x=0$. Then there exists a unique weak solution $(u, n, p, \varphi)$ to the problem (1.1)-(1.8) satisfying

$$
\begin{aligned}
& (n, p) \in L^{\infty}(0, T ; L \log L) \cap L^{2}\left(Q_{T}\right) \cap L^{4 / 3}\left(0, T ; W^{1, \frac{4}{3}}\right), \quad n, p \geq 0 \quad \text { in } \quad Q_{T}, \\
& \left(n_{t}, p_{t}\right) \in L^{4 / 3}\left(0, T ;\left(W^{1,4}\right)^{*}\right), \\
& \varphi \in L^{\infty}\left(0, T ; H^{1}\right) \cap L^{2}\left(0, T ; H^{2}\right), \quad \nabla \varphi \in L^{4}\left(Q_{T}\right), \\
& u \in V_{2}\left(Q_{T}\right):=L^{\infty}\left(0, T ; L^{2}\right) \cap L^{2}\left(0, T ; H^{1}\right) \subset L^{4}\left(Q_{T}\right), \\
& u_{t} \in L^{4 / 3}\left(0, T ; H^{-1}\right) .
\end{aligned}
$$

REMARK 1.2. We point out that the existence part of Theorem 1.1 is a slight modification of the Theorem 1 in Ryham [15], where the author considered the same system, but with the following boundary condition:

$$
u=0, \quad(\nabla n-n \nabla \varphi) \cdot \nu=(\nabla p+p \nabla \varphi) \cdot \nu=\varphi=0 \quad \text { on } \partial \Omega .
$$

Here $\nu$ is the unit outward normal to the boundary $\partial \Omega$. However, our method could not be generalized to this case.

Theorem 1.3. Let $\left(n_{0}, p_{0}\right) \in L^{3 / 2}, u_{0} \in L^{2}, n_{0}, p_{0} \geq 0$ in $\Omega \subseteq \mathbb{R}^{3}$ and $\int_{\Omega} \varphi \mathrm{d} x=0$.

Assume further that

$$
u \in L^{s}\left(0, T ; L^{q, \infty}(\Omega)\right) \quad \text { with } \quad \frac{2}{s}+\frac{3}{q}=1, \quad 3<q \leq \infty .
$$

Here $L^{p, \infty}(\Omega) \equiv L_{w}^{p}(\Omega)$ denotes the standard Lorentz space [16]. Then there exists a 
unique weak solution $(u, n, p, \varphi)$ to the problem (1.1)-(1.8) satisfying

$$
\begin{aligned}
& \left(n^{\frac{3}{4}}, p^{\frac{3}{4}}\right) \in V_{2}\left(Q_{T}\right) \subset L^{10 / 3}\left(Q_{T}\right), \quad n, p \geq 0 \quad \text { in } \quad Q_{T}, \\
& (n, p) \in L^{\infty}\left(0, T ; L^{3 / 2}\right) \cap L^{5 / 2}\left(Q_{T}\right) \cap L^{5 / 3}\left(0, T ; W^{1,5 / 3}\right) \cap L^{4}\left(0, T ; L^{2}\right), \\
& \left(n_{t}, p_{t}\right) \in L^{5 / 3}\left(0, T ;\left(W^{1,3}\right)^{*}\right), \\
& \varphi \in L^{\infty}\left(0, T ; W^{2,3 / 2}\right) \cap L^{5 / 2}\left(0, T ; W^{2,5 / 2}\right), \\
& \nabla \varphi \in L^{\infty}\left(0, T ; L^{3}\right) \cap L^{5 / 2}\left(0, T ; L^{15}\right), \\
& u \in V_{2}\left(Q_{T}\right), \quad u_{t} \in L^{2}\left(0, T ;\left(W^{1,3}\right)^{*}\right) .
\end{aligned}
$$

REMARK 1.4. Our result is new for equations (1.3), (1.4) and (1.5) when formally setting $u \equiv 0$, and hence (1.10) holds. When (1.10) holds, S. Dubois [17] proved the uniqueness of weak solutions to the Navier-Stokes Equations (1.1), (1.2) when $\varphi \equiv 0$.

REMARK 1.5. The existence part of Theorem 1.3 can be proved by the Galerkin method and thus we omit the details here. We only need to derive the estimates (1.9) and (1.11) and prove the uniqueness.

Our proof will be be based on carefully using the low regularity estimates by introducing two auxiliary functions $N$ and $P$ (see (2.10) and (2.11)).

Let $p \in[0,+\infty]$ and $q \in[0,+\infty]$. We define the Lorentz space

$$
L^{p, q}(\Omega):=\left\{f: \Omega \rightarrow \mathcal{R}, \text { measureable } \mid\|f\|_{L^{p, q}}<+\infty\right\},
$$

where

$$
\|f\|_{L^{p, q}}:= \begin{cases}\left(\frac{q}{p} \int_{0}^{+\infty}\left(t^{\frac{1}{p}} f^{*}(t)\right)^{q} \frac{d t}{t}\right)^{\frac{1}{q}} & q<+\infty, \\ \sup _{t>0} \frac{1}{t^{p}} f^{*}(t), & q=+\infty .\end{cases}
$$

Here

$$
\begin{aligned}
& f^{*}(t):=\inf \left\{s>0 \mid \lambda_{f}(s) \leq t\right\} \\
& \lambda_{f}(t):=\operatorname{mes}\{|f|>s\} .
\end{aligned}
$$

It is well known that

$$
L^{p, p} \equiv L^{p}
$$

We will use the following generalized Hölder inequality [16]:

$$
\|f g\|_{L^{p, q}} \leq C\|f\|_{L^{p_{1}, q_{1}}}\|g\|_{L^{p_{2}, q_{2}}},
$$

with $\frac{1}{p}=\frac{1}{p_{1}}+\frac{1}{p_{2}}$ and $\frac{1}{q}=\frac{1}{q_{1}}+\frac{1}{q_{2}}$. We will also use the following notation:

$$
\operatorname{LLog} L(\Omega):=\left\{f\left|\int_{\Omega}\right| f \mid \log (e+|f|) d x<+\infty\right\}
$$




\section{Proofs of Theorem 1.1}

First, we recall a technical lemma due to D. Chae [18].

Lemma 2.1 (18, Lem. 2.1)). For any bounded domain $\Omega \subseteq \mathbb{R}^{2}$ with a smooth boundary we have a continuous imbedding

$$
L \log L(\Omega) \hookrightarrow\left(H^{1}(\Omega)\right)^{*} .
$$

Lemma 2.2. $n, p \geq 0$ in $Q_{T}$, and

$$
\begin{gathered}
\int_{\Omega} n \log n+p \log p \mathrm{~d} x+4 \int_{0}^{T} \int_{\Omega}(\nabla \sqrt{n})^{2}+(\nabla \sqrt{p})^{2} \mathrm{~d} x \mathrm{~d} t \\
+\varepsilon \int_{0}^{T} \int_{\Omega}(\Delta \varphi)^{2} \mathrm{~d} x \mathrm{~d} t=\int_{\Omega} n_{0} \log n_{0}+p_{0} \log p_{0} \mathrm{~d} x \\
\nabla \varphi \in L^{\infty}\left(0, T ; L^{2}\right) .
\end{gathered}
$$

Proof. By the maximum principle, it is easy to prove that $n \geq 0$ and $p \geq 0$ in $Q_{T}$.

Testing (1.3) by $1+\log n$ and testing (1.4) by $1+\log p$ respectively, and using the divergence free property, summing up the resulting equality, we easily get (2.2).

Testing (1.5) by $\varphi$, we see that

$$
\begin{aligned}
\varepsilon\|\nabla \varphi\|_{L^{2}}^{2} & =-\int_{\Omega}(n-p) \varphi \mathrm{d} x \leq\|n-p\|_{\left(H^{1}(\Omega)\right)^{*}}\|\varphi\|_{H^{1}} \\
& \leq C\|n-p\|_{L \log L}\|\varphi\|_{H^{1}} \quad(\text { by }(2.1)) \\
& \leq C\|\nabla \varphi\|_{L^{2}} .
\end{aligned}
$$

Here, we used the Poincaré inequality

$$
\left\|\varphi-\int_{\Omega} \varphi \mathrm{d} x\right\|_{L^{2}} \leq C\|\nabla \varphi\|_{L^{2}}
$$

and the assumption $\int_{\Omega} \varphi \mathrm{d} x=0$.

This proves $(2.3)$.

LEMMA 2.3.

$$
u \in V_{2}\left(Q_{T}\right) \subset L^{4}\left(Q_{T}\right) .
$$

Proof. Testing (1.1) by $u$ and taking into account the divergence-free property, we find that

$$
\begin{aligned}
\frac{1}{2} \frac{d}{d t} \int_{\Omega} u^{2} \mathrm{~d} x+\int_{\Omega}|\nabla u|^{2} \mathrm{~d} x & =\varepsilon \int_{\Omega} \Delta \varphi \nabla \varphi \cdot u \mathrm{~d} x \\
& \leq \varepsilon\|\Delta \varphi\|_{L^{2}}\|\nabla \varphi\|_{L^{4}}\|u\|_{L^{4}} \\
& \leq \varepsilon\|\Delta \varphi\|_{L^{2}}^{2}+\varepsilon\|\nabla \varphi\|_{L^{4}}^{2}\|u\|_{L^{4}}^{2} \\
& \leq \varepsilon\|\Delta \varphi\|_{L^{2}}^{2}+C \varepsilon\|\nabla \varphi\|_{L^{4}}^{2}\|u\|_{L^{2}}\|\nabla u\|_{L^{2}} \\
& \leq \frac{1}{2}\|\nabla u\|_{L^{2}}^{2}+\varepsilon\|\Delta \varphi\|_{L^{2}}^{2}+C \varepsilon\|\nabla \varphi\|_{L^{4}}^{4}\|u\|_{L^{2}}^{2},
\end{aligned}
$$


and by the Gagliardo-Nirenberg inequality

$$
\|v\|_{L^{4}}^{2} \leq C\|v\|_{L^{2}}\|\nabla v\|_{L^{2}}, \text { for any } v \in H_{0}^{1}(\Omega) .
$$

Gronwall's inequality gives (2.5).

LEMMA 2.4.

$$
\begin{gathered}
(n, p) \in L^{2}\left(Q_{T}\right) \cap L^{4 / 3}\left(0, T ; W^{1,4 / 3}\right), \\
\left(n_{t}, p_{t}\right) \in L^{4 / 3}\left(0, T ;\left(W^{1,4}\right)^{*}\right), \\
u_{t} \in L^{4 / 3}\left(0, T ; H^{-1}\right) .
\end{gathered}
$$

Proof. By the parabolic Sobolev imbedding theorem $V_{2}\left(Q_{T}\right) \subset L^{4}\left(Q_{T}\right)$, it is easy to obtain $(n, p) \in L^{2}\left(Q_{T}\right)$. Since $\nabla n=2 \nabla \sqrt{n} \cdot \sqrt{n}, \nabla \sqrt{n} \in L^{2}\left(Q_{T}\right), \sqrt{n} \in L^{4}\left(Q_{T}\right)$, we easily infer that $\nabla n \in L^{4 / 3}\left(Q_{T}\right)$ by the Hölder inequality.

This proves (2.7) by a similar calculations for $p$.

It is standard to prove (2.8) and (2.9) and thus we omit the details here.

Now we are in a position to prove the uniqueness. Let $\left(u_{i}, \pi_{i}, n_{i}, p_{i}, \varphi_{i}\right)(i=1,2)$ be two weak solutions to the problem (1.1)-(1.8). Also let us denote

$$
u:=u_{1}-u_{2}, \quad \pi:=\pi_{1}-\pi_{2}, \quad n:=n_{1}-n_{2}, \quad p:=p_{1}-p_{2}, \quad \varphi:=\varphi_{1}-\varphi_{2} .
$$

Now we can define $N$ and $P$ satisfying the following equations

$$
\left\{\begin{array}{l}
\int_{\Omega} N \mathrm{~d} x=0 \\
-\Delta N=n \text { in } \Omega \\
\left.\frac{\partial N}{\partial \nu}\right|_{\partial \Omega}=0
\end{array}\right.
$$

and

$$
\left\{\begin{array}{l}
\int_{\Omega} P \mathrm{~d} x=0 \\
-\Delta P=p \text { in } \Omega \\
\left.\frac{\partial P}{\partial \nu}\right|_{\partial \Omega}=0
\end{array}\right.
$$

From (1.5), (2.10), and (2.11), we know that

$$
\varepsilon \varphi=-N+P .
$$

It is easy to infer that

$$
n_{t}+\nabla \cdot\left(u_{1} n+u n_{2}\right)=\Delta n-\nabla \cdot\left(n \nabla \varphi_{1}+n_{2} \nabla \varphi\right) .
$$

Testing this equation by $N$, we obtain

$$
\begin{gathered}
\frac{1}{2} \frac{d}{d t} \int_{\Omega}|\nabla N|^{2} \mathrm{~d} x+\int_{\Omega}|\Delta N|^{2} \mathrm{~d} x=\int_{\Omega} n \nabla \varphi_{1} \cdot \nabla N+n_{2} \nabla \varphi \cdot \nabla N+u_{1} n \cdot \nabla N \\
+u n_{2} \cdot \nabla N \mathrm{~d} x=: I_{1}+I_{2}+I_{3}+I_{4} .
\end{gathered}
$$


Each term $I_{i}(i=1,2,3,4)$ can be bounded as follows.

$$
\begin{aligned}
I_{1} & \leq\|\Delta N\|_{L^{2}}\|\nabla N\|_{L^{4}}\left\|\nabla \varphi_{1}\right\|_{L^{4}} \\
& \leq C\|\Delta N\|_{L^{2}}^{3 / 2}\|\nabla N\|_{L^{2}}^{\frac{1}{2}}\left\|\nabla \varphi_{1}\right\|_{L^{4}} \quad(\text { by }(2.6)) \\
& \leq \frac{1}{8}\|\Delta N\|_{L^{2}}^{2}+C\left\|\nabla \varphi_{1}\right\|_{L^{4}}^{4}\|\nabla N\|_{L^{2}}^{2}, \\
I_{2} & \leq\left\|n_{2}\right\|_{L^{2}}\|\nabla \varphi\|_{L^{4}}\|\nabla N\|_{L^{4}} \\
& \left.\leq C\left\|n_{2}\right\|_{L^{2}}\left(\|\nabla N\|_{L^{4}}+\|\nabla P\|_{L^{4}}\right)\|\nabla N\|_{L^{4}} \quad \quad \text { by }(2.12)\right) \\
& \leq C\left\|n_{2}\right\|_{L^{2}}\left(\|\nabla N\|_{L^{4}}^{2}+\|\nabla P\|_{L^{4}}^{2}\right) \\
& \leq C\left\|n_{2}\right\|_{L^{2}}\|\nabla N\|_{L^{2}}\|\Delta N\|_{L^{2}}+C\left\|n_{2}\right\|_{L^{2}}\|\nabla P\|_{L^{2}}\|\Delta P\|_{L^{2}} \\
& \leq \frac{1}{8}\|\Delta N\|_{L^{2}}^{2}+C\left\|n_{2}\right\|_{L^{2}}^{2}\|\nabla N\|_{L^{2}}^{2}+\frac{1}{8}\|\Delta P\|_{L^{2}}^{2}+C\left\|n_{2}\right\|_{L^{2}}^{2}\|\nabla P\|_{L^{2}}^{2}, \\
I_{3} & \leq\left\|u_{1}\right\|_{L^{4}}\|\Delta N\|_{L^{2}}\|\nabla N\|_{L^{4}} \\
& \leq C\left\|u_{1}\right\|_{L^{4}}\|\Delta N\|_{L^{2}}^{\frac{3}{2}}\|\nabla N\|_{L^{2}}^{\frac{1}{2}} \quad(\text { by }(2.6)) \\
& \leq \frac{1}{8}\|\Delta N\|_{L^{2}}^{2}+C\left\|u_{1}\right\|_{L^{4}}^{4}\|\nabla N\|_{L^{2}}^{2}, \\
I_{4} & \leq\left\|n_{2}\right\|_{L^{2}}\|u\|_{L^{4}}\|\nabla N\|_{L^{4}} \\
& \leq\left\|n_{2}\right\|_{L^{2}}\|\nabla N\|_{L^{4}}^{2}+\left\|n_{2}\right\|_{L^{2}}\|u\|_{L^{4}}^{2} \\
& \leq C\left\|n_{2}\right\|_{L^{2}}\|\nabla N\|_{L^{2}}\|\Delta N\|_{L^{2}}+C\left\|n_{2}\right\|_{L^{2}}\|u\|_{L^{2}}\|\nabla u\|_{L^{2}} \\
& \leq \frac{1}{8}\|\Delta N\|_{L^{2}}^{2}+\frac{1}{8}\|\nabla u\|_{L^{2}}^{2}+C\left\|n_{2}\right\|_{L^{2}}^{2}\|\nabla N\|_{L^{2}}^{2}+C\left\|n_{2}\right\|_{L^{2}}^{2}\|u\|_{L^{2}}^{2} .
\end{aligned}
$$

Substituting these estimates into (2.13), we find that

$$
\begin{aligned}
& \frac{1}{2} \frac{d}{d t} \int_{\Omega}(\nabla N)^{2} \mathrm{~d} x+\frac{1}{2} \int_{\Omega}|\Delta N|^{2} \mathrm{~d} x \\
\leq & C\left(\left\|\nabla \varphi_{1}\right\|_{L^{4}}^{4}+\left\|n_{2}\right\|_{L^{2}}^{2}+\left\|u_{1}\right\|_{L^{4}}^{4}\right)\|\nabla N\|_{L^{2}}^{2} \\
& +\frac{1}{8}\|\Delta P\|_{L^{2}}^{2}+C\left\|n_{2}\right\|_{L^{2}}^{2}\|\nabla P\|_{L^{2}}^{2}+\frac{1}{8}\|\nabla u\|_{L^{2}}^{2}+C\left\|n_{2}\right\|_{L^{2}}^{2}\|u\|_{L^{2}}^{2} .
\end{aligned}
$$

Similarly for the $p$-equation, we obtain

$$
\begin{aligned}
& \frac{1}{2} \frac{d}{d t} \int_{\Omega}|\nabla P|^{2} \mathrm{~d} x+\frac{1}{2} \int_{\Omega}|\Delta P|^{2} \mathrm{~d} x \\
\leq & C\left(\left\|\nabla \varphi_{1}\right\|_{L^{4}}^{4}+\left\|p_{2}\right\|_{L^{2}}^{2}+\left\|u_{1}\right\|_{L^{4}}^{4}\right)\|\nabla P\|_{L^{2}}^{2} \\
& \quad+\frac{1}{8}\|\Delta N\|_{L^{2}}^{2}+C\left\|p_{2}\right\|_{L^{2}}^{2}\|\nabla N\|_{L^{2}}^{2}+\frac{1}{8}\|\nabla u\|_{L^{2}}^{2}+C\left\|p_{2}\right\|_{L^{2}}^{2}\|u\|_{L^{2}}^{2} .
\end{aligned}
$$

It is easy to find that $u$ satisfies

$$
u_{t}+u_{1} \cdot \nabla u+u \cdot \nabla u_{2}+\nabla \pi=\Delta u+\varepsilon \Delta \varphi \nabla \varphi_{1}+\varepsilon \Delta \varphi_{2} \nabla \varphi .
$$

Testing this equation by $u$ and using the divergence-free property, we obtain

$$
\begin{aligned}
\frac{1}{2} \frac{d}{d t} \int_{\Omega} u^{2} \mathrm{~d} x+\int_{\Omega}|\nabla u|^{2} \mathrm{~d} x & =\int_{\Omega} \varepsilon \Delta \varphi \nabla \varphi_{1} \cdot u+\varepsilon \Delta \varphi_{2} \nabla \varphi \cdot u-(u \cdot \nabla) u_{2} \cdot u \mathrm{~d} x \\
& =: J_{1}+J_{2}+J_{3},
\end{aligned}
$$


Each term $J_{i}(i=1,2,3)$ can be bounded as follows.

$$
\begin{aligned}
J_{1} \leq & \varepsilon\|\Delta \varphi\|_{L^{2}}\left\|\nabla \varphi_{1}\right\|_{L^{4}}\|u\|_{L^{4}} \\
& \leq \frac{1}{8}\|\Delta N\|_{L^{2}}^{2}+\frac{1}{8}\|\Delta P\|_{L^{2}}^{2}+C\left\|\nabla \varphi_{1}\right\|_{L^{4}}^{2}\|u\|_{L^{4}}^{2} \\
\leq & \left.\frac{1}{8}\|\Delta N\|_{L^{2}}^{2}+\frac{1}{8}\|\Delta P\|_{L^{2}}^{2}+\frac{1}{8}\|\nabla u\|_{L^{2}}^{2}+C\left\|\nabla \varphi_{1}\right\|_{L^{4}}^{4}\|u\|_{L^{2}}^{2} \quad \text { (by }(2.6)\right) \\
J_{2} \leq & \varepsilon\left\|\Delta \varphi_{2}\right\|_{L^{2}}\|\nabla \varphi\|_{L^{4}}\|u\|_{L^{4}} \\
\leq & \varepsilon\left\|\Delta \varphi_{2}\right\|_{L^{2}}\left(\|\nabla N\|_{L^{4}}+\|\nabla P\|_{L^{4}}\right)\|u\|_{L^{4}} \quad(\text { by }(2.12)) \\
\leq & \varepsilon\left\|\Delta \varphi_{2}\right\|_{L^{2}}\left(\|\nabla N\|_{L^{4}}^{2}+\|\nabla P\|_{L^{4}}^{2}\right)+\varepsilon\left\|\Delta \varphi_{2}\right\|_{L^{2}}\|u\|_{L^{4}}^{2} \\
\leq & \frac{1}{8}\|\Delta N\|_{L^{2}}^{2}+\frac{1}{8}\|\Delta P\|_{L^{2}}^{2}+\frac{1}{8}\|\nabla u\|_{L^{2}}^{2} \\
& +C\left\|\Delta \varphi_{2}\right\|_{L^{2}}^{2}\left(\|\nabla N\|_{L^{2}}^{2}+\|\nabla P\|_{L^{2}}^{2}+\|u\|_{L^{2}}^{2}\right) \\
J_{3} \leq & \|u\|_{L^{4}}^{2}\left\|\nabla u_{2}\right\|_{L^{2}} \leq C\|u\|_{L^{2}}\|\nabla u\|_{L^{2}}\left\|\nabla u_{2}\right\|_{L^{2}} \\
\leq & \frac{1}{8}\|\nabla u\|_{L^{2}}^{2}+C\left\|\nabla u_{2}\right\|_{L^{2}}^{2}\|u\|_{L^{2}}^{2} .
\end{aligned}
$$

Inserting the above estimate into (2.16), we arrive at

$$
\begin{aligned}
& \frac{1}{2} \frac{d}{d t} \int_{\Omega} u^{2} \mathrm{~d} x+\frac{5}{8} \int_{\Omega}|\nabla u|^{2} \mathrm{~d} x \leq \frac{1}{4}\|\Delta N\|_{L^{2}}^{2}+\frac{1}{4}\|\Delta P\|_{L^{2}}^{2} \\
& \quad+C\left(\left\|\nabla \varphi_{1}\right\|_{L^{4}}^{4}+\left\|\Delta \varphi_{2}\right\|_{L^{2}}^{2}+\left\|\nabla u_{2}\right\|_{L^{2}}^{2}\right)\|u\|_{L^{2}}^{2} \\
& \quad+C\left\|\Delta \varphi_{2}\right\|_{L^{2}}^{2}\left(\|\nabla N\|_{L^{2}}^{2}+\|\nabla P\|_{L^{2}}^{2}\right) .
\end{aligned}
$$

Adding up (2.14), (2.15), and (2.17) we conclude that

$$
\frac{d}{d t} \int_{\Omega}|\nabla N|^{2}+|\nabla P|^{2}+|u|^{2} \mathrm{~d} x \leq C(t) \int_{\Omega}|\nabla N|^{2}+|\nabla P|^{2}+|u|^{2} \mathrm{~d} x
$$

with some $C(t) \in L^{1}(0, T)$. Gronwall's inequality gives $N=0=P=u$ and thus $n=p=$ $0=\varphi$, which completes the proof.

\section{Proof of Theorem 1.2}

First, we derive the estimates (1.11).

LEMMA 3.1.

$$
\begin{gathered}
\int_{\Omega} n^{\frac{3}{2}}+p^{\frac{3}{2}} \mathrm{~d} x+4 \int_{0}^{T} \int_{\Omega}\left(\nabla n^{\frac{3}{4}}\right)^{2}+\left(\nabla p^{\frac{3}{4}}\right)^{2} \mathrm{~d} x \mathrm{~d} t \\
+\frac{1}{2 \varepsilon} \int_{0}^{T} \int_{\Omega}(n-p)\left(n^{\frac{3}{2}}-p^{\frac{3}{2}}\right) \mathrm{d} x \mathrm{~d} t=\int_{\Omega} n_{0}^{\frac{3}{2}}+p_{0}^{\frac{3}{2}} \mathrm{~d} x, \\
(n, p) \in L^{\infty}\left(0, T ; L^{\frac{3}{2}}\right) \cap L^{\frac{5}{2}}\left(Q_{T}\right) \cap L^{\frac{5}{3}}\left(0, T ; W^{1,5 / 3}\right) \cap L^{4}\left(0, T ; L^{2}\right) .
\end{gathered}
$$

Proof. Testing (1.3), (1.4) by $\sqrt{n}, \sqrt{p}$, respectively, using the divergence-free property, and summing up the resulting equation, we obtain (3.1).

From (3.1), it follows easily that

$$
\left(n^{\frac{3}{4}}, p^{\frac{3}{4}}\right) \in V_{2}\left(Q_{T}\right) \subset L^{\frac{10}{3}}\left(Q_{T}\right)
$$


and thus

$$
(n, p) \in L^{\infty}\left(0, T ; L^{\frac{3}{2}}\right) \cap L^{\frac{5}{2}}\left(Q_{T}\right) .
$$

On the other hand, $\nabla n=\frac{4}{3}\left(\nabla n^{\frac{3}{4}}\right) \cdot n^{\frac{1}{4}}, \nabla n^{\frac{3}{4}} \in L^{2}\left(Q_{T}\right), n^{\frac{1}{4}} \in L^{10}\left(Q_{T}\right)$, thus $\nabla n \in$ $L^{\frac{5}{3}}\left(Q_{T}\right)$ by the Hölder's inequality.

By the interpolation inequality

$$
\|n\|_{L^{2}} \leq\|n\|_{L^{\frac{3}{2}}}^{\frac{3}{8}}\|n\|_{L^{\frac{5}{2}}}^{\frac{5}{8}}
$$

we obtain

$$
n \in L^{4}\left(0, T ; L^{2}\right)
$$

This proves (3.2).

LEMMA 3.2.

$$
\begin{gathered}
\varphi \in L^{\infty}\left(0, T ; W^{2, \frac{3}{2}}\right) \cap L^{5 / 2}\left(0, T ; W^{2,5 / 2}\right) \cap L^{4}\left(0, T ; H^{2}\right), \\
\varphi \in L^{\infty}\left(0, T ; W^{1,3}\right) \cap L^{5 / 2}\left(0, T ; W^{1,15}\right) .
\end{gathered}
$$

Proof. By the standard regularity theory of elliptic second order equations and (3.2), we easily obtain (3.3) and (3.4) follows from the Sobolev embedding theorem $W^{2, \frac{3}{2}} \subset W^{1,3}$ and $W^{2, \frac{5}{2}} \subset W^{1,15}$.

LEMMA 3.3.

$$
\begin{gathered}
u \in V_{2}\left(Q_{T}\right) \subset L^{10 / 3}\left(Q_{T}\right), \\
u_{t} \in L^{2}\left(0, T ;\left(W^{1,3}\right)^{*}\right), \quad\left(n_{t}, p_{t}\right) \in L^{5 / 3}\left(0, T ;\left(W^{1,3}\right)^{*}\right) .
\end{gathered}
$$

Proof. Testing (1.1) by $u$ and using (1.2), we see that

$$
\begin{aligned}
\frac{1}{2} \frac{d}{d t} \int_{\Omega} u^{2} \mathrm{~d} x+\int_{\Omega}|\nabla u|^{2} \mathrm{~d} x & =\int_{\Omega} \varepsilon \Delta \varphi \nabla \varphi \cdot u \mathrm{~d} x \\
& \leq \varepsilon\|\Delta \varphi\|_{L^{2}}\|\nabla \varphi\|_{L^{6}}\|u\|_{L^{3}} \\
& \leq C\|\varphi\|_{H^{2}}^{2}\|\nabla u\|_{L^{2}} \leq \frac{1}{2}\|\nabla u\|_{L^{2}}^{2}+C\|\varphi\|_{H^{2}}^{4} .
\end{aligned}
$$

Using (3.3) and Gronwall's inequality, we obtain (3.5).

The proof of (3.6) is standard and thus we omit the details here.

Now we are in a position to prove the uniqueness. We still use the same notation as in section 2, and similarly we get (2.13). But each term $I_{i}(i=1,2,3,4)$ can be bounded as follows

$$
\begin{aligned}
I_{1} & \leq\|\Delta N\|_{L^{2}}\|\nabla N\|_{L^{\frac{30}{13}}}\left\|\nabla \varphi_{1}\right\|_{L^{15}} \\
& \leq C\|\Delta N\|_{L^{2}}^{\frac{6}{5}}\|\nabla N\|_{L^{2}}^{\frac{5}{5}}\left\|\nabla \varphi_{1}\right\|_{L^{15}} \\
& \leq \frac{1}{8}\|\Delta N\|_{L^{2}}^{2}+C\left\|\nabla \varphi_{1}\right\|_{L^{15}}^{\frac{5}{2}}\|\nabla N\|_{L^{2}}^{2}
\end{aligned}
$$


and by the Gagliardo-Nirenberg inequality

$$
\begin{gathered}
\|\nabla N\|_{L^{\frac{30}{13}}} \leq C\|\nabla N\|_{L^{2}}^{\frac{4}{5}}\|\Delta N\|_{L^{2}}^{\frac{1}{5}} . \\
I_{2} \leq\left\|n_{2}\right\|_{L^{2}}\|\nabla \varphi\|_{L^{6}}\|\nabla N\|_{L^{3}} \leq C\left\|n_{2}\right\|_{L^{2}}\left(\|\Delta N\|_{L^{2}}+\|\Delta P\|_{L^{2}}\right)\|\nabla N\|_{L^{3}} \\
\leq C\left\|n_{2}\right\|_{L^{2}}\left(\|\Delta N\|_{L^{2}}+\|\Delta P\|_{L^{2}}\right)\|\nabla N\|_{L^{2}}^{\frac{1}{2}}\|\Delta N\|_{L^{2}}^{\frac{1}{2}} \\
\leq \frac{1}{8}\|\Delta N\|_{L^{2}}^{2}+\frac{1}{8}\|\Delta P\|_{L^{2}}^{2}+C\left\|n_{2}\right\|_{L^{2}}^{4}\|\nabla N\|_{L^{2}}^{2},
\end{gathered}
$$

and by the Gagliardo-Nirenberg inequality

$$
\begin{gathered}
\|\nabla N\|_{L^{3}}^{2} \leq C\|\nabla N\|_{L^{2}}\|\Delta N\|_{L^{2}} . \\
I_{3} \leq C\left\|u_{1}\right\|_{L^{q, \infty}}\|\Delta N\|_{L^{2}}\|\nabla N\|_{L^{\frac{2 q}{q-2}, 2}} \quad(\operatorname{by}(1.12)) \\
\leq C\left\|u_{1}\right\|_{L^{q, \infty}}\|\Delta N\|_{L^{2}}^{1+\frac{3}{q}}\|\nabla N\|_{L^{2} \frac{3}{q}}^{1-\frac{3 q}{8}} \\
\leq \frac{1}{8}\|\Delta N\|_{L^{2}}^{2}+C\left\|u_{1}\right\|_{L^{q, \infty}}^{\frac{2 q}{q-3}}\|\nabla N\|_{L^{2}}^{2},
\end{gathered}
$$

and by the Gagliardo-Nivenberg inequality ([16])

$$
\begin{gathered}
\|\nabla N\|_{L^{\frac{2 q}{q-2}, 2}} \leq C\|\nabla N\|_{L^{2}}^{1-\frac{3}{q}}\|\Delta N\|_{L^{2}}^{\frac{3}{q}}, \quad q>3 . \\
I_{4} \leq\left\|n_{2}\right\|_{L^{2}}\|u\|_{L^{6}}\|\nabla N\|_{L^{3}} \leq C\left\|n_{2}\right\|_{L^{2}}\|\nabla u\|_{L^{2}}\|\nabla N\|_{L^{2}}^{\frac{1}{2}}\|\Delta N\|_{L^{2}}^{\frac{1}{2}} \quad(\operatorname{by}(3.8)) \\
\leq \frac{1}{8}\|\nabla u\|_{L^{2}}^{2}+\frac{1}{8}\|\Delta N\|_{L^{2}}^{2}+C\left\|n_{2}\right\|_{L^{2}}^{4}\|\nabla N\|_{L^{2}}^{2} .
\end{gathered}
$$

Inserting the above estimates into (2.13), we get

$$
\begin{aligned}
& \frac{1}{2} \frac{d}{d t} \int_{\Omega}|\nabla N|^{2} \mathrm{~d} x+\frac{1}{2} \int_{\Omega}|\Delta N|^{2} \mathrm{~d} x \\
\leq & C\left(\left\|\nabla \varphi_{1}\right\|_{L^{15}}^{5 / 2}+\left\|n_{2}\right\|_{L^{2}}^{4}+\left\|u_{1}\right\|_{L^{q, \infty}}^{\frac{2 q}{q-3}}\right)\|\nabla N\|_{L^{2}}^{2}+\frac{1}{8}\|\Delta P\|_{L^{2}}^{2}+\frac{1}{8}\|\nabla u\|_{L^{2}}^{2} .
\end{aligned}
$$

Similarly for the $p$-equation, we obtain

$$
\begin{aligned}
& \frac{1}{2} \frac{d}{d t} \int_{\Omega}|\nabla P|^{2} \mathrm{~d} x+\frac{1}{2} \int_{\Omega}|\Delta P|^{2} \mathrm{~d} x \\
\leq & C\left(\left\|\nabla \varphi_{1}\right\|_{L^{15}}^{5 / 2}+\left\|p_{2}\right\|_{L^{2}}^{4}+\left\|u_{1}\right\|_{L^{q, \infty}}^{\frac{2 q}{q-3}}\right)\|\nabla N\|_{L^{2}}^{2}+\frac{1}{8}\|\Delta N\|_{L^{2}}^{2}+\frac{1}{8}\|\nabla u\|_{L^{2}}^{2} .
\end{aligned}
$$

As in section 2, we have (2.16).

Each term $J_{i}(i=1,2,3)$ can be bounded as follows:

$$
\begin{aligned}
J_{1} & \leq \varepsilon\left\|\nabla \varphi_{1}\right\|_{L^{15}}\|\Delta \varphi\|_{L^{2}}\|u\|_{L^{\frac{30}{13}}} \\
& \leq C\left\|\nabla \varphi_{1}\right\|_{L^{15}}\left(\|\Delta N\|_{L^{2}}+\|\Delta P\|_{L^{2}}\right)\|u\|_{L^{2}}^{\frac{4}{5}}\|\nabla u\|_{L^{2}}^{\frac{1}{5}} \quad(\text { by }(3.7)) \\
& \leq \frac{1}{8}\|\Delta N\|_{L^{2}}^{2}+\frac{1}{8}\|\Delta P\|_{L^{2}}^{2}+\frac{1}{8}\|\nabla u\|_{L^{2}}^{2}+C\left\|\nabla \varphi_{1}\right\|_{L^{15}}^{5 / 2}\|u\|_{L^{2}}^{2},
\end{aligned}
$$




$$
\begin{aligned}
J_{2} & \leq \varepsilon\left\|\Delta \varphi_{2}\right\|_{L^{2}}\|\nabla \varphi\|_{L^{6}}\|u\|_{L^{3}} \\
& \leq C\left\|n_{2}+p_{2}\right\|_{L^{2}}\left(\|\Delta N\|_{L^{2}}+\|\Delta P\|_{L^{2}}\right)\|u\|_{L^{2}}^{\frac{1}{2}}\|\nabla u\|_{L^{2}}^{\frac{1}{2}} \quad(\operatorname{by}(3.8)) \\
& \leq \frac{1}{8}\|\Delta N\|_{L^{2}}^{2}+\frac{1}{8}\|\Delta P\|_{L^{2}}^{2}+\frac{1}{8}\|\nabla u\|_{L^{2}}^{2}+C\left\|n_{2}+p_{2}\right\|_{L^{2}}^{4}\|u\|_{L^{2}}^{2}, \\
J_{3} & =\int_{\Omega}(u \cdot \nabla) u \cdot u_{2} \mathrm{~d} x \leq C\left\|u_{2}\right\|_{L^{q, \infty}}\|\nabla u\|_{L^{2}}\|u\|_{L^{\frac{2 q}{q-2}, 2}} \quad(\operatorname{by}(1.12)) \\
& \leq C\left\|u_{2}\right\|_{L^{q, \infty}}\|\nabla u\|_{L^{2}}^{1+\frac{3}{q}}\|u\|_{L^{2}}^{1-\frac{3}{q}} \quad(\operatorname{by}(3.9)) \\
& \leq \frac{1}{8}\|\nabla u\|_{L^{2}}^{2}+C\left\|u_{2}\right\|_{L^{q, \infty}}^{\frac{2 q}{q-3}}\|u\|_{L^{2}}^{2} .
\end{aligned}
$$

Substituting the above estimates into (2.16), we obtain

$$
\begin{aligned}
& \frac{1}{2} \frac{d}{d t} \int_{\Omega} u^{2} \mathrm{~d} x+\frac{5}{8} \int_{\Omega}|\nabla u|^{2} \mathrm{~d} x \\
\leq & \frac{1}{4}\|\Delta N\|_{L^{2}}^{2}+\frac{1}{4}\|\Delta P\|_{L^{2}}^{2}+C\left(\left\|\nabla \varphi_{1}\right\|_{L^{15}}^{5 / 2}+\left\|n_{2}+p_{2}\right\|_{L^{2}}^{4}+\left\|u_{2}\right\|_{L^{q, \infty}}^{\frac{2 q}{q-3}}\right)\|u\|_{L^{2}}^{2} .
\end{aligned}
$$

Adding up (3.10), (3.11), and (3.12), we arrive at

$$
\frac{d}{d t} \int_{\Omega}|\nabla N|^{2}+|\nabla P|^{2}+|u|^{2} \mathrm{~d} x \leq C(t) \int_{\Omega}|\nabla N|^{2}+|\nabla P|^{2}+|u|^{2} \mathrm{~d} x
$$

with some $C(t) \in L^{1}(0, T)$. Gronwall's inequality gives $N=P=0, u=0$ and hence $n=p=\varphi=0$, which proves $u_{1}=u_{2}, n_{1}=n_{2}, p_{1}=p_{2}, \varphi_{1}=\varphi_{2}$.

The proof is completed.

Acknowledgement. This work supported partially supported by a China NSF Grant No. 10871097, National Basic Research Program of China (973 Program) No. 2007CB814800, China Postdoctoral Research Foundation No. 20080441062 and Jiangsu Planned Projects for Postdoctoral Research Foundation No. 0802020C.

The authors are indebted to the two referees for their careful reading of the manuscript, pointing out a new reference [15], and many nice suggestions which improved the presentation of the paper.

\section{REFERENCES}

[1] M. Bazant and T. Squires, Induced-charge electro-kinetic phenomena, Theory and Microfluidic Applications, Phys. Rev. Lett., 92, 066101, 2004.

[2] Y. Ben, E.A. Demekhin and H.C. Chang, A spectral theory for small amplitude miscible fingering, Phys of Fluids, 14, 999-1010, 2002.

[3] R.E. Problestein, Physicochemical Hydrodynamics, An Introduction, John Wiley and Sons, INC., 1994.

[4] K. Chu and M. Bazant, Electrochemical thin films at and above the classical limiting current, SIAM J. Appl. Math., 65, 1485-1505, 2005.

[5] I. Rubinstein, Electro-Diffusion of Ions, SIAM, Philadelphia, 1990.

[6] M. Kurokiba and T. Ogawa, $L^{p}$ well-posedness for the drift-diffusion system arising from the semiconductor device simulation, J. Math. Anal. Appl., 342, 1052-1067, 2008.

[7] P. Biler, W. Hebisch and T. Nadzieja, The Debye system: existence and large time behavior of solutions, Nonlinear Anal. TMA, 23, 1189-1209, 1994.

[8] W. Fang and K. Ito, Global solutions of the time-dependent drift-diffusion semiconductor equations, J. Diff. Equations, 123, 523-566, 1995.

[9] H. Gajewski, Gröger K., On the basic equations for carrier transport in semiconductors, J. Math. Anal. Appl., 113, 12-35, 1986.

[10] T. Squires and M. Bazant, Induced-charge electro-osmosis, J. Fluid Mech., 509, 217-252, 2004. 
[11] H. Stone and S. Kim, Microfluidics: basic issues, applications, and challenges, J. AICHE, 47, 1250-1254, 2001.

[12] S. Thamida and H.C. Chang, Nonlinear electro-kinetic ejection and entrainment due to polarization at nearly insulated wedges, Phys Fluids, 14, 4315-4328, 2002.

[13] R. Ryham, C. Liu and ZQ. Wang, Mathematical modelling for electro-kinetic fluids: analysis and simulation, Preprint, 2005.

[14] R.Ryham, C. Liu and Z.Q. Wang, On electro-kinetic fluids: one dimensional configurations, Discrete and Continuous Dynamical Systems-series B, 6, 357-371, 2006.

[15] R. Ryham, Global existence and long term behavior of $2 D$ electro-hydrodynamics, arXiv: 0810.2064 v1 [math.AP] 12 Oct 2008.

[16] H. Triebel, Theory of Function Spaces II, Birkhäuser Basel, 1992.

[17] S. Dubois, Uniqueness for some Leray-Hopf solutions to the Navier-Stokes equations, J. Diff. Equations, 189, 99-147, 2003.

[18] D. Chae, Weak solutions of 2-D incompressible Euler equations, Nonlinear Anal. TMA, 23, 629-638, 1994. 